\title{
Compositional Effects and Mechanical Parametric Analysis of Outwash Deposits Based on the Randomised Generation of Stone Blocks
}

\author{
Chong Shi ${ }^{1,2}$ and Jinzhou Bai ${ }^{1,2}$ \\ ${ }^{1}$ Key Laboratory of Ministry of Education for Geomechanics and Embankment Engineering, Hohai University, Nanjing 210098, China \\ ${ }^{2}$ Institute of Geotechnical Engineering, Hohai University, Nanjing 210098, China \\ Correspondence should be addressed to Chong Shi; shichong81@126.com
}

Received 17 September 2014; Accepted 25 November 2014

Academic Editor: Zhaohui Li

Copyright (C) 2015 C. Shi and J. Bai. This is an open access article distributed under the Creative Commons Attribution License, which permits unrestricted use, distribution, and reproduction in any medium, provided the original work is properly cited.

\begin{abstract}
Based on the distribution of the stone blocks in outwash deposits, the paper present a modeling method for the random structure of outwash deposits, in which the long axis of the stone blocks is supposed obeying a lognormal distribution. Then numerical experiments of biaxial compression using the granular discrete element method are used in the macro- and micro parametric analysis. The influences of strength of the cementation, the sizes of stone blocks, and the content of stone blocks on the peak compressive and shear strength are discussed. The micromechanical parameters of the outwash deposits are also analyzed. The proposed method offers a supplement to the mechanical characterization of outwash deposits and accounts for the limitation that indoor experiments cannot consider large stone blocks.
\end{abstract}

\section{Introduction}

Outwash deposits are a particular type of geological medium and are formed by glacial movement. The media are composed primarily of irregular stone blocks with high strength and weak, fine-grained debris, as shown in Figure 1. They are widely distributed in southwestern China, where the water resources are rich and the geological conditions are suitable for the construction of hydropower plants. According to the program "accelerating the development of hydropower project in China," outwash deposits should be an important and difficult issue and will receive more attention from the departments of geological engineering, geotechnical engineering, and hydropower engineering. However, due to the composition of outwash deposits, the mechanical parameters of outwash deposits are difficult to be determined by current analysis methods, as well as reinforcement measures.

Outwash deposits are different from common homogeneous materials because of the presence of stone blocks and the cementation of fine-grained debris. For any structural characteristics and material composition, the mechanical properties and the water-physical properties of them are unique. At present, most studies consider the outwash deposit as a homogeneous medium. The analytical approaches rely mainly on indoor experiments and numerical simulations. However, because large stone blocks cannot be used in the indoor experiments, the mechanical characterization of outwash deposits has some limitations. Some scholars $[1,2]$ found that the existence of boulders increased the strength and density of outwash deposits, which also leads to deviations in their mechanical properties. Others studied the relationships among the cementation of fine-grained debris, dry density, water content, block shapes, block distribution, and the shear strength of the stone and debris mixture by experimental tests, such as the large triaxial shear experiment, the medium-sized triaxial shear experiment, and the large horizontal push shear experiment [3-7]. The results demonstrate that the stress-strain of this medium is approximately taken on nonlinear hardening characteristics, which agrees well with the hyperbola assumption of DuncanChang model, and the shear strength of the stone and debris mixture is related closely to the concentration and distribution of the stone blocks. However, it is difficult to gain a thorough understanding of outwash deposits without 


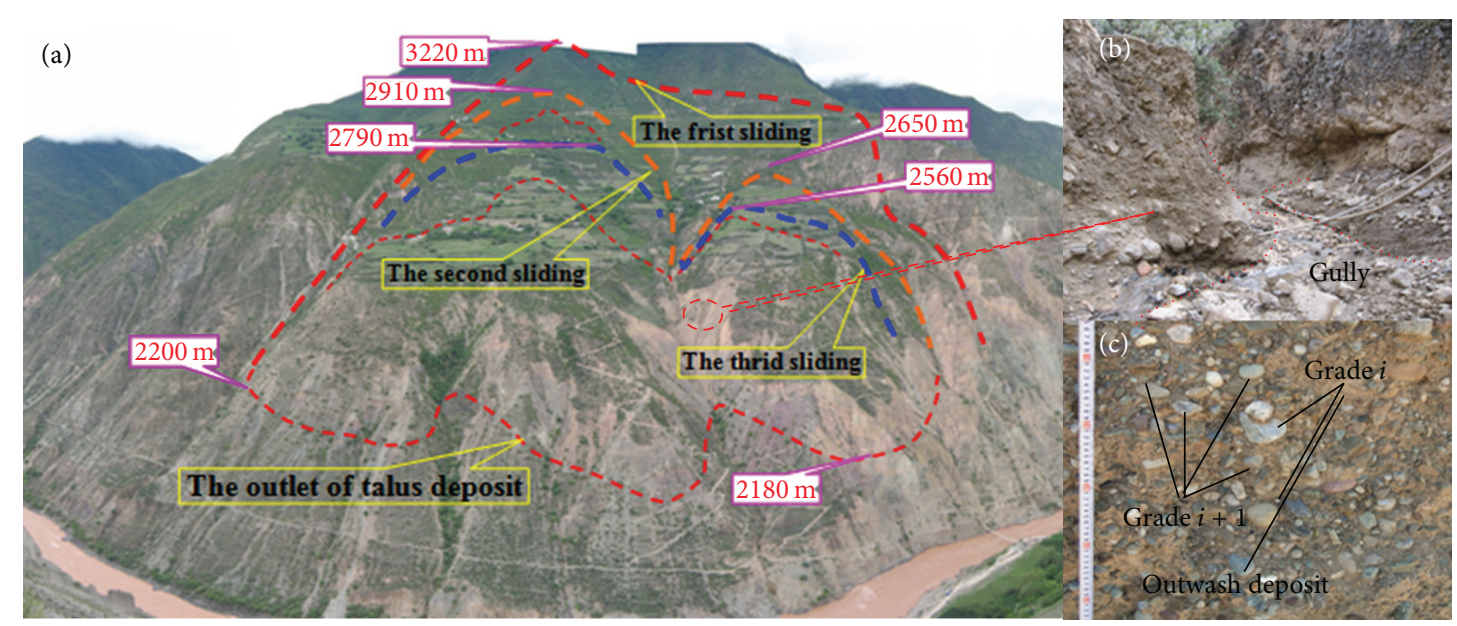

Figure 1: A typical outwash deposit formed by glacial movement.

considering the effects of heterogeneity and randomness on the distribution and concentration of stone blocks in the conventional geotechnical experiments. The limited field tests are discrete and not representative. Therefore, it is necessary to consider the composition of the outwash deposit in the experiments and numerical calculations when studying the mechanical characteristics. Digital image processing is a convenient tool. The sizes and shapes of the debris in outwash deposits were studied by Lebourg et al. [8]. The real internal structure of geological materials was also detected by such technology [9-12]. So it can provide an effective approach for research into the composition of outwash deposits $[13,14]$. Because the structure composition of outwash deposits is mainly determined by stone blocks, a model considering the distribution and randomness of stone blocks is meaningful.

According to the field statistics, the geometric shapes of the stone blocks are assumed to be approximately ellipticpolygon and obey a normal distribution in this work. Then a geomechanical model for biaxial compression is established using the particle flow code. The effects of the stone blocks and fine-grained debris on the mechanical properties of outwash deposit are studied. The changes in the macroscopic compressive strength and shear strength of the medium are also discussed.

\section{Structural Characteristics of Outwash Deposits}

2.1. The Structural Composition of Outwash Deposits. Outwash deposits are a type of binary mixture medium consisting of fine-grained debris and stone blocks, where both the debris and stone blocks are made of the same material and are distinguished by the grain size but not by the composition. However, not all sizes of stone blocks have a positive influence on the strength of the medium. There is a threshold that distinguishes debris from stone blocks. The threshold size of stone blocks can be determined only in certain geological conditions and scales, which shows that the large grains play a greater role in the macroscopic mechanical properties during the smaller scale studies. When the scale of research is increased, the former large grains will lose their dominant effect to mechanical property and can be regarded as debris. The following criterion to distinguish stones from debris is proposed [15]:

$$
f= \begin{cases}R & d \geq d_{\mathrm{thr}} \\ S & d<d_{\mathrm{thr}}\end{cases}
$$

where $d_{\mathrm{thr}}$ is the threshold of stone and debris $\left(d_{\mathrm{thr}}=0.05 L_{c}\right)$, $L_{c}$ is the characteristic engineering size, $R$ denotes the stone, and $S$ denotes the debris.

In addition, the indoor geotechnical test on similar media, the direct shear test, and triaxial compression test can only consider grains below $2 \mathrm{~cm}$ due to the limitations of the instruments. Therefore, $d_{\mathrm{thr}}$ is set to be $2 \mathrm{~cm}$ in this work, which means that if the grain size is less than $2 \mathrm{~cm}$, the grain will be defined as debris. Conversely, if the grain size is greater than $2 \mathrm{~cm}$, the grain will be considered as a stone block.

By above measure, grains of debris and stone blocks will be endowed attributes separately to reflect the change rule of medium composition and mechanical property.

2.2. The Aggregate Gradation of Outwash Deposits. The sizes and shapes of stone blocks differ greatly at different part, as shown in Figure 1(c). The microstructure of outwash deposits, which can be explained as grain contact and interaction, is just related to content, size, and roughness of stone blocks. In order to construct a stochastic model of outwash deposits, the grains regarded as stone can be divided into several gradation according to the grain size. Then according to the results of the grain size analysis and field statistics, the proportion, quality, volume, and area of the stone blocks in the research region can be obtained easily. The grains smaller than the threshold will be considered as debris and can be described using the conventional geotechnical experiments. Numerical simulation method will be used to reflect its influence on mechanical property of outwash deposit, in which the stone blocks are supposed to be unbroken. 


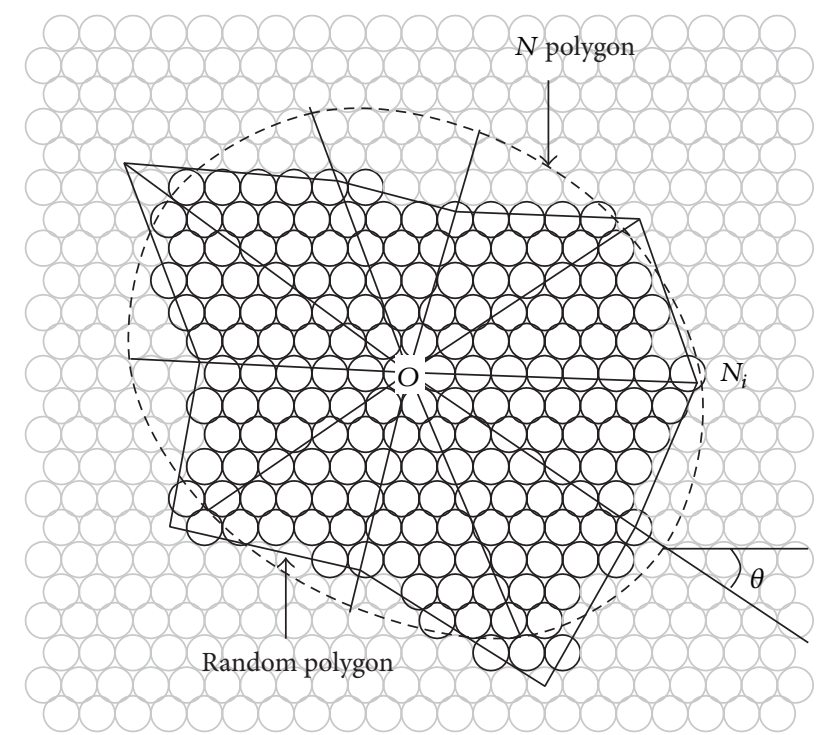

FIGURE 2: An arbitrarily shaped stone block depicted by a particles cluster.

If the density of the stone blocks is $\rho_{R}$, the debris density is $\rho_{S}$. Therefore, the $2 \mathrm{D}$ area of the stone blocks in grade $i$ can be written as follows:

$$
A_{i}=\frac{4 r^{2} G_{i} / \rho_{R}}{G_{0} / \rho_{S}+\sum_{k=1}^{n} G_{i} / \rho_{R}},
$$

where $A_{i}$ is the $2 \mathrm{D}$ area percentage of stone blocks in grade $i, G_{0}$ is the weight of the medium with grain sizes below the threshold and $\sum_{k=1}^{n} G_{k} / \rho_{R}$ is the total weight of stone blocks.

2.3. The Randomised Generation of Aggregates. Each stone block can be regarded as an arbitrary polygon in the plane, so a number of polygons are generated randomly in the designated region to simulate blocks. During the generation process, every polygon should be judged to avoid overlapping with others. Then the area in the polygons is regarded as stone blocks of different grades. When the polygon is convex, it has difficulty in considering the interaction force due to mosaic effect of rough block surface, which will lead to deviations in the mechanical parameter research, so the polygon generation should consider convexity and concavity at the same time.

To generate the arbitrary shapes of the stone blocks, the stone blocks are assumed to be initially elliptical, then the outline is divided as polygon and the vertices of polygon are random, as shown in Figure 2. The ellipses can be determined using three parameters: the length of long axis $a$, length of the short axis $b$ (or the axis ratio defined as ratio of the short axis length to the long axis), and the azimuth of the long axis $\theta$. Field investigation shows that the angle of long axis azimuth is in poor statistical correlation, so it can be regarded as obeying uniform distribution over $0 \sim 360^{\circ}$.

As shown in Figure 3(a), the lengths of the long axis and the axis ratio are approximately obeying normal distribution with variance as shown in Figures 3(c) and 3(d). So the length of the long axis, the axial ratio, and the azimuth angle of long axis whose definition is shown in Figure 3(b) are given as shown in the following formula:

$$
\begin{gathered}
a=a_{0}+2 \sigma_{a}(\operatorname{rand}(1)-1), \\
r=r_{0}+2 \sigma_{r}(\operatorname{rand}(1)-1), \\
\theta=360 \cdot \operatorname{rand}(1),
\end{gathered}
$$

where $a, \sigma_{a}, r, \sigma_{r}$ are the mean of the long axis, the variance of the long axis, the mean of the axial ratio, and the variance of axial ratio, respectively; $\theta$ is the azimuth of long axis. rand(1) returns a random number between 0 and 1. $a, \sigma_{a}$, $r, \sigma_{r}$ should be obtained using the statistics according to the stone blocks in outwash deposit medium. Here, 0.5 times the corresponding mean value is used as an empirical parameter for variance if there is no statistical information.

Then, every stone block is hypothesised to be a polygon composed of $N$ sides as shown in Figure 2. The elliptical arc is accordingly divided into $N$ arcs. Each point of the arc can be moved towards the centre of the ellipse. The corresponding coordinate of each point can be defined as follows:

$$
\begin{gathered}
x_{i}=x_{0}+a \cdot \cos (\theta+N \Delta \varphi), \\
y_{i}=y_{0}+a \cdot r \cdot \sin (\theta+N \Delta \varphi),
\end{gathered}
$$

where $x_{i}$ and $y_{i}$ are the coordinates of the $i$ th node in a polygon, $x_{0}$ and $y_{0}$ are the coordinates of the centre of the ellipse, $\varphi_{0}$ is the azimuth of ellipse, and $\Delta \varphi$ is the angle between two vertexes of the polygon where $\Delta \varphi=360 / N$.

The steps of the random structure generation are shown in Figure 4. The generated polygons can be convex or concave. The polygons are divided into $n$ triangles using the centre and vertexes of the polygon. Therefore, once the centre of an element is determined to be within one triangle, the element can be set as a stone block and numbered.

To ensure that the stone blocks do not overlap, the elements are set as debris by default. Once one of the elements in the current trial has been defined as a stone block, the current step will end and a new position will be tried as the centre of the generated polygon until all of the elements in the generated polygon are no longer debris. When all of the generated polygons are finished in this way, the numerical model is complete.

To avoid setting adjacent polygons as the same stone block, each stone block should be numbered a unique number. The particle contact parameters within one stone block are endowed the parameters of the stone blocks, and the contact parameter between two stone blocks is taken as that of debris. Therefore, the contact characteristics between stone blocks can be approximately simulated.

The content rate of stone block of grade $i$ in a twodimension model can be defined as the ratio of the stone block area within grade $i$ to the total area of the model. It can be expressed as follows:

$$
n_{i}=\frac{\sum A_{j}}{A}
$$



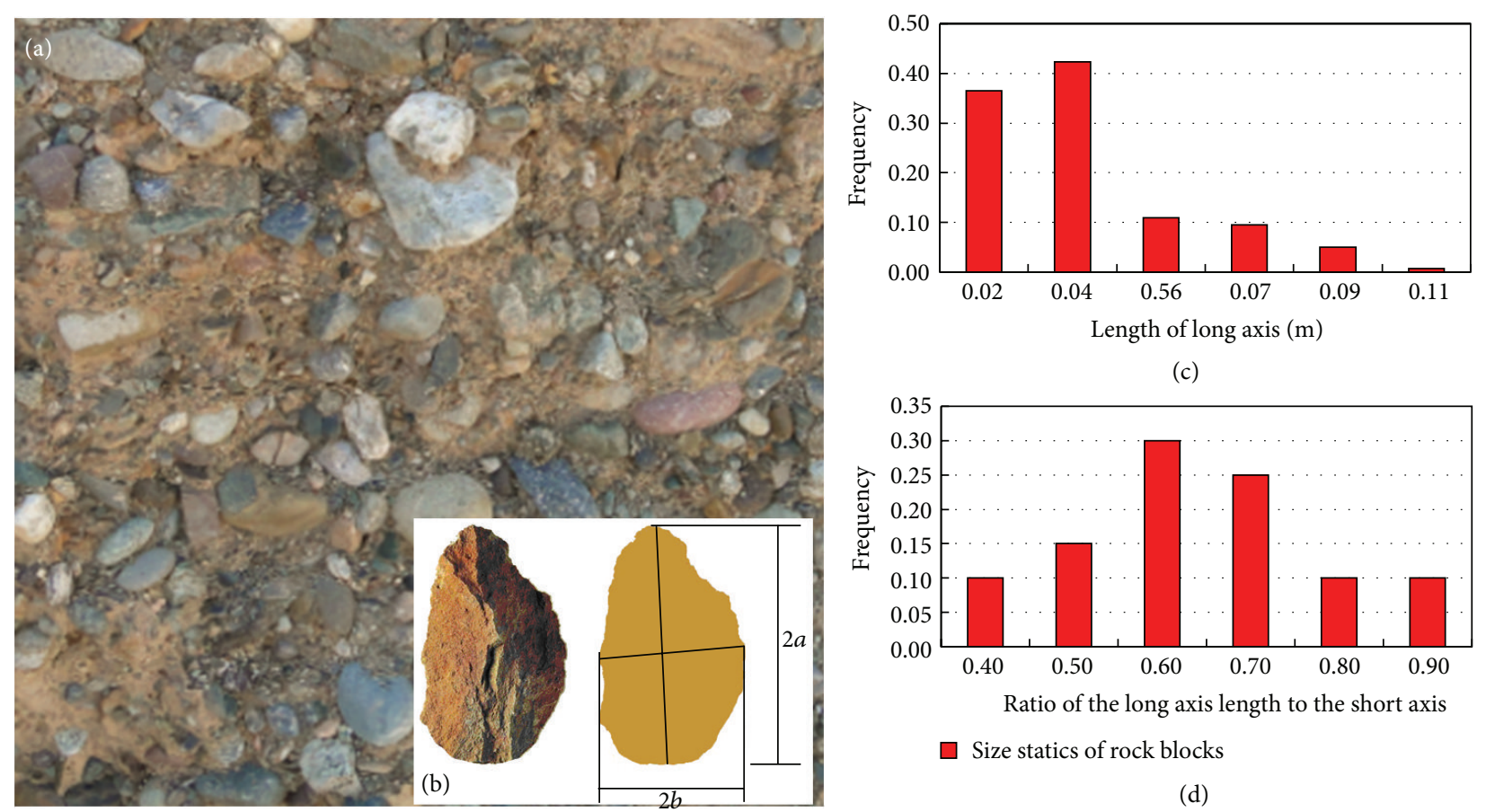

(c)

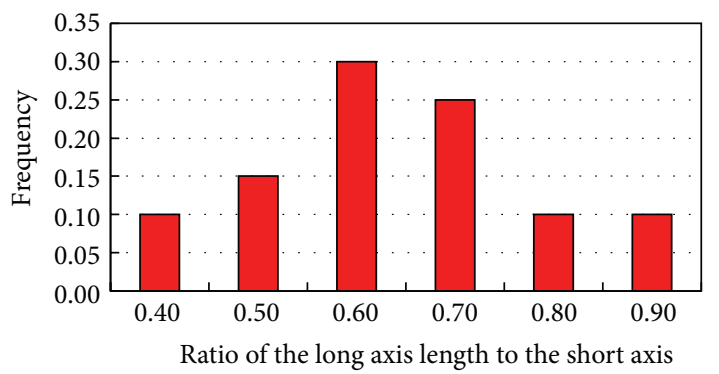

$\square$ Size statics of rock blocks

(d)

Figure 3: The statistical results of the size and the lengths of the long axis of stone blocks. (a) the in situ outwash deposits; (b) definition of the long axis and short axis for stone blocks; the long axis is the largest distance between vertices along the stone outline, while the short axis is the maximum segment length normal to the long axis direction; (c) the statistical results of the size of stone blocks; (d) the statistical results of the lengths of the long axis of stone blocks.

where $A$ is the total area of the model, $A_{j}$ is the area of the $j$ th stone block and grade $i$, and $n_{i}$ is the content rate of stone blocks of grade $i$.

The area of the total stone blocks is counted until the grade requirement is met.

The structure of the outwash deposit model is generated randomly according to the method of multigradations. The influences of the azimuth, concavity, and convexity of the stone blocks on the outwash deposit are also taken into consideration. The generation of stone blocks occurs from the largest to the smallest size according to the grain size accumulation curve.

\section{Analysis Based on Micromechanical Model}

3.1. The Principle of the Granular Discrete Element Method. The granular discrete element method is adopted to simulate the micromechanical properties of the granular materials in the form of discs or spherical particles using the explicit difference algorithm [16]. Newton's Second Law is used in order to determine the particle movement that is caused by contact or the force of a body, while the force-displacement law is used to calculate the contact force that is changing relative to movement. The two laws are then used alternately until the total system reaches equilibrium as shown in Figure 5(a). The particles can be combined into any shape or combination to simulate the stone or debris mass structures.

As shown in Figure 5(b), when there is no bonding between particles, the mechanical behaviour of the particles in contact is determined by their own normal stiffness $\left(k_{n}\right)$, tangential stiffness $\left(k_{s}\right)$, and friction coefficient $(\mu)$. When two particles are in contact, the contact and tangential stiffness can be calculated as follows:

$$
\begin{aligned}
& k_{n}=\frac{k_{n}^{(A)} k_{n}^{(B)}}{k_{n}^{(A)}+k_{n}^{(B)}}, \\
& k_{s}=\frac{k_{s}^{(A)} k_{s}^{(B)}}{k_{s}^{(A)}+k_{s}^{(B)}} .
\end{aligned}
$$

The normal force of contact particles is depicted as

$$
F_{n}=K_{n} U_{n}
$$

where $U_{n}$ is the normal contact depth.

In the calculation process, the tangential force is zero initially, so the incremental form of the equation is used as follows:

$$
\Delta F_{s}=-k_{s} \Delta U_{s}
$$

If $U_{n} \leq 0$, a gap exists between the particles, the normal and shear force are both zero, and the slipping force between the particles should be calculated with the friction coefficient. This coefficient is calculated as $\mu=\min \left(\mu^{(A)}, \mu^{(B)}\right)$, where $\mu^{(A)}$ and $\mu^{(B)}$ are the particle friction coefficients of $A$ and $B$, respectively, and $F_{s}=\mu F_{n}$ is used when $F_{s} \geq \mu F_{n}$.

The stiffness contact model is adopted for the outwash deposit in this work because the point contact and the 


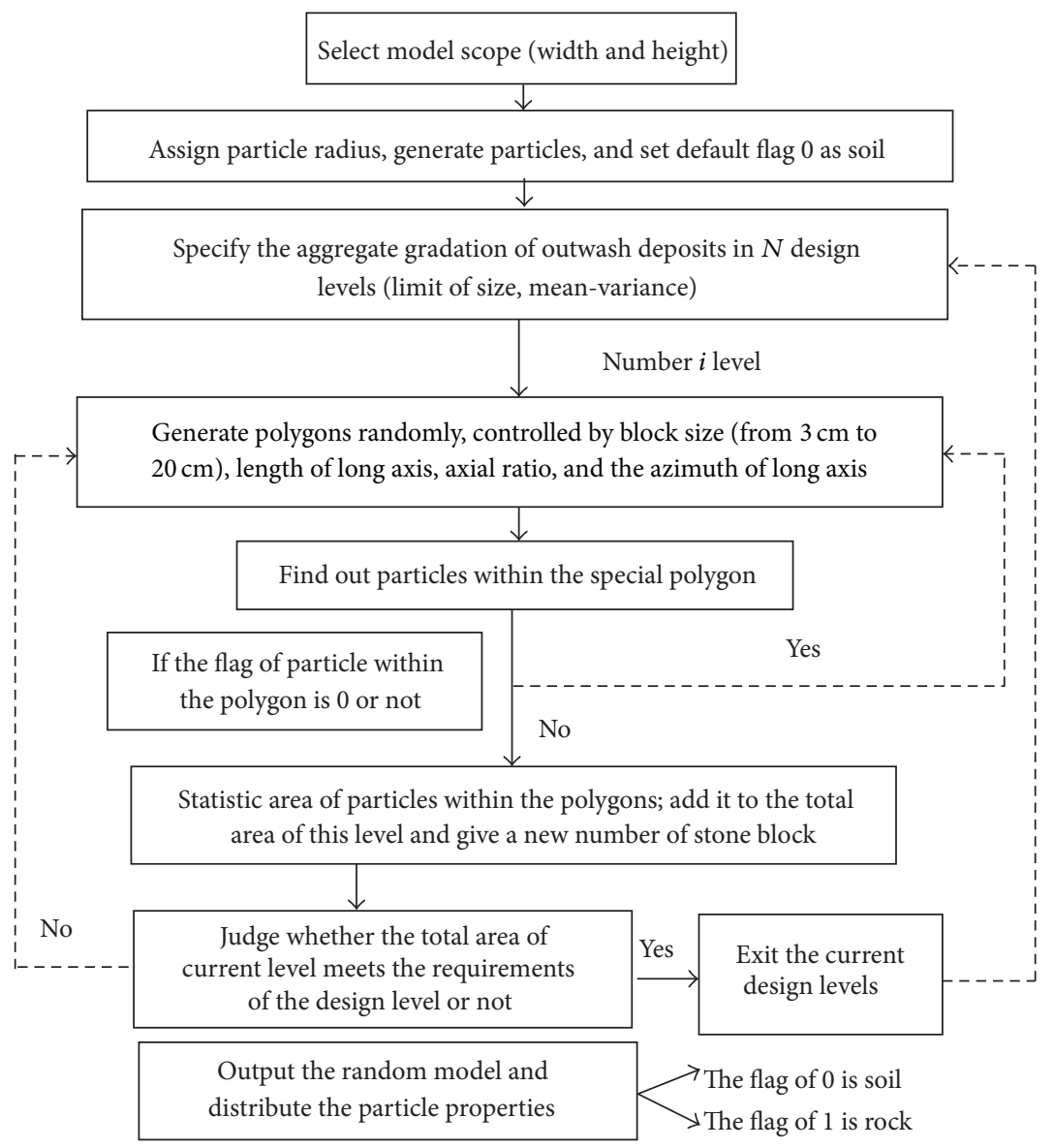

FIgURE 4: The flowchart for the randomised generation of stone blocks.

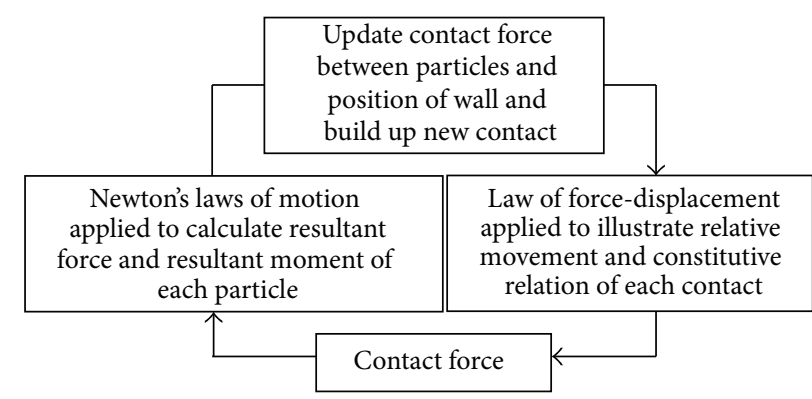

(a) Calculation principle in PFC2D

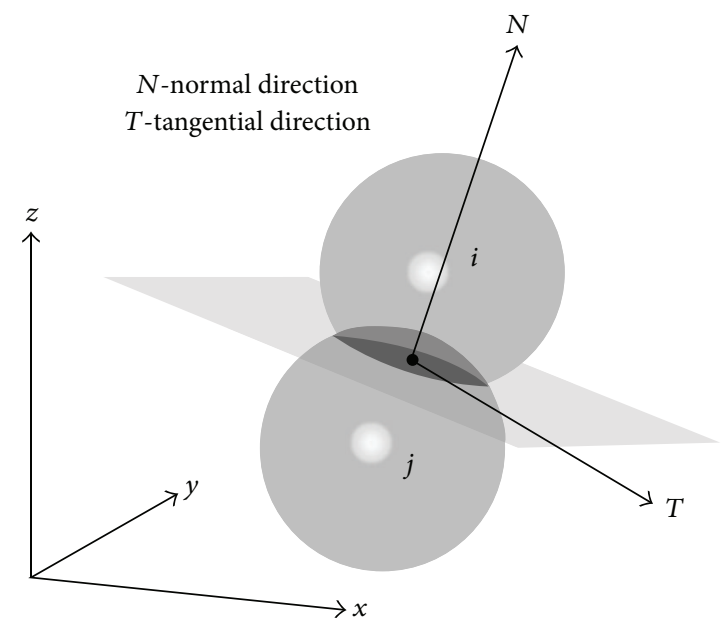

(b) Two contacting particles

FIGURE 5: Contact force between particles and calculation principle.

linear contact in the debris-debris, debris-stone, and stonestone are the main kind of contact types; the point contacts are especially dominant. Five microparameters need to be calibrated: the normal stiffness, the stiffness ratio of tangent stiffness and normal stiffness, the friction coefficient, the normal bonding strength, and the tangent bonding strength according to PFC2D manual [17].

3.2. The Conversion from the Geological Model to a Mechanical Model. According to the theory of granular discrete element 

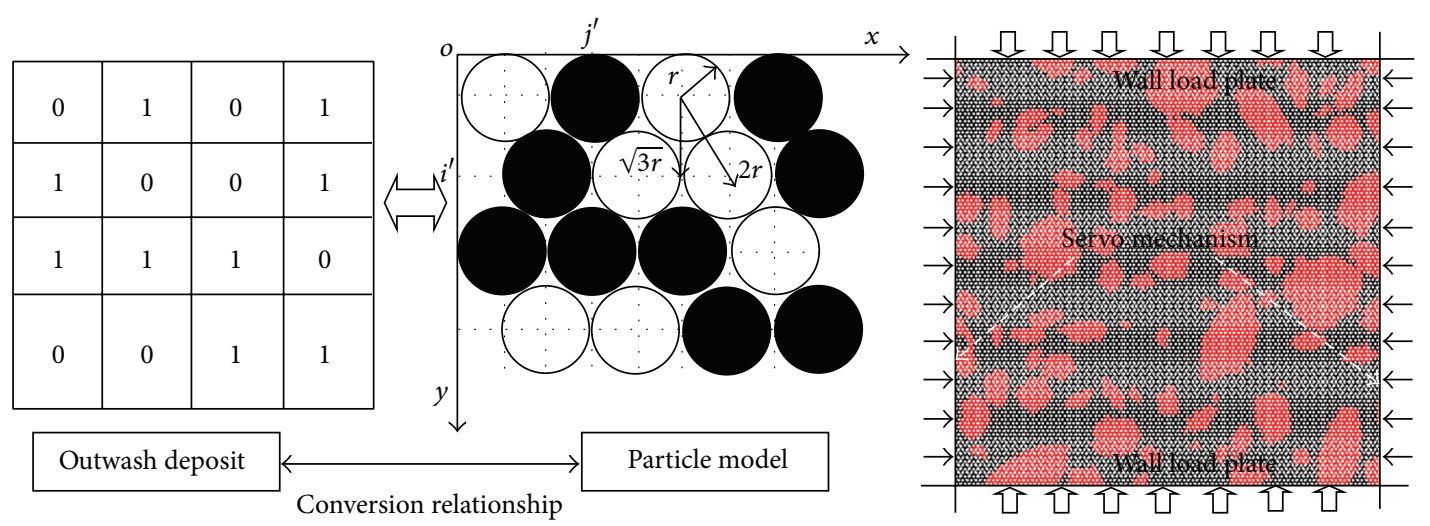

FIGURE 6: Construction of a debris-stone micromodel using a spot matrix and PFC2D.

method, 2D disk particles are used to transform the geological model to a mechanical model. If the particle is too large in the model, the shape of stone block and microstructure characteristic will be distorted. However, the larger number of particles in a model will lead to very slow calculation speed. A significant amount of time will be sacrificed, which may not be tolerable. So a proper particle size should be determined empirically.

The width and the height of the model in this work are taken to $0.50 \times 0.50 \mathrm{~m}$, the constant radius of particles is $2.5 \mathrm{~mm}$, and the arrangement of the particles is shown in Figure 6 . The distance between the centres of two adjacent particles in the vertical direction is $\sqrt{3} r$ ( $r$ is the radius of particle). The distance between the centres of adjacent particles in the horizontal direction is $2 r$. Therefore, the coordinates of the particle centre $\left(x_{i^{\prime}, j^{\prime}}, y_{i^{\prime}, j^{\prime}}\right)$ in the $i^{\prime}$ line and $j^{\prime}$ column can be expressed as follows:

$$
\begin{gathered}
x_{i^{\prime}, j^{\prime}}= \begin{cases}\left(2 j^{\prime}-1\right) r & \left(i^{\prime}=2 k+1\right), \quad(k \in N), \\
2 j^{\prime} r & \left(i^{\prime}=2 k\right),\end{cases} \\
y_{i^{\prime}, j^{\prime}}=r+\sqrt{3} r(i-1) .
\end{gathered}
$$

According to the modelling steps, the particles are arranged initially as shown in Figure 6. The even rows are dislocated to ensure that the particles are tangential with adjacent rows. When a particle model filling the research zone is generated, every particle within the model will be judged to determine whether its centre is located in some random polygon. If it is in a polygon, the particle belongs to a stone block with a certain flag. If it is not in any polygon, the particle is regarded as debris. The initial structural information can be obtained from site research or by random generation.

Several particles with the same polygon flag are assembled together by the high bonding force to simulate the characteristics of the stone blocks. The structural effect of the outwash deposit is thus simulated. The separation of the debris from the stone is also convenient for the determination of the mechanical parameters.

3.3. The Relationship between the Macro- and Micromechanical Parameters. Young's modulus and Poisson's ratio

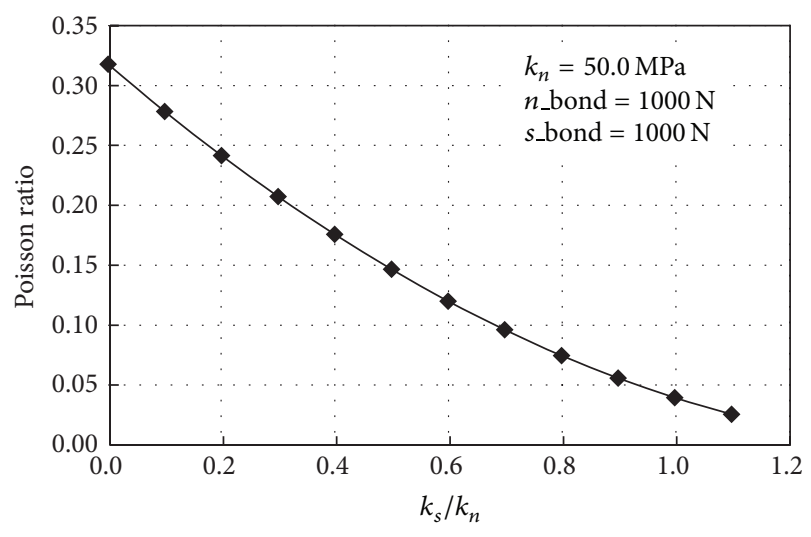

FIgure 7: The relationship between Poisson's ratio and the stiffness ratio.

determined in the indoor geotechnical tests are used to describe the macrodeformation properties of the materials. Many studies have shown that Young's modulus is related to the microparameter $k_{n}$ and the stiffness ratio $k_{s} / k_{n}$, while Poisson's ratio is mainly related to the stiffness ratio [18, 19]. However, these changes are all affected by the particle compositions, such as the mean radius and the ratio of the maximum radius to the minimum radius. Since a constant radius is used in this work, the problem can be neglected.

To reflect the relation between Poisson's ratio and the stiffness ratio, $k_{s} / k_{n}$, the normal stiffness is initially fixed at a constant $50 \mathrm{MPa}$. The relationship between Poisson's ratio and the stiffness ratio is shown in Figure 7. It can be found that Poisson's ratio in the debris shows a nonlinear, inverse relationship with the stiffness ratio of debris when the particle radius is unchanged.

It can also be concluded in Figure 7 that the tangent stiffness is usually less than the normal stiffness. Once the tangent stiffness is equal to or greater than the normal stiffness, Poisson's ratio will reach zero. This situation only exists in very loose cellular structures. However, for outwash deposits, it is obviously unreasonable when the medium is in a compacted state. Poisson's ratio declines as the stiffness ratio increases. When the tangent stiffness is equal to the 


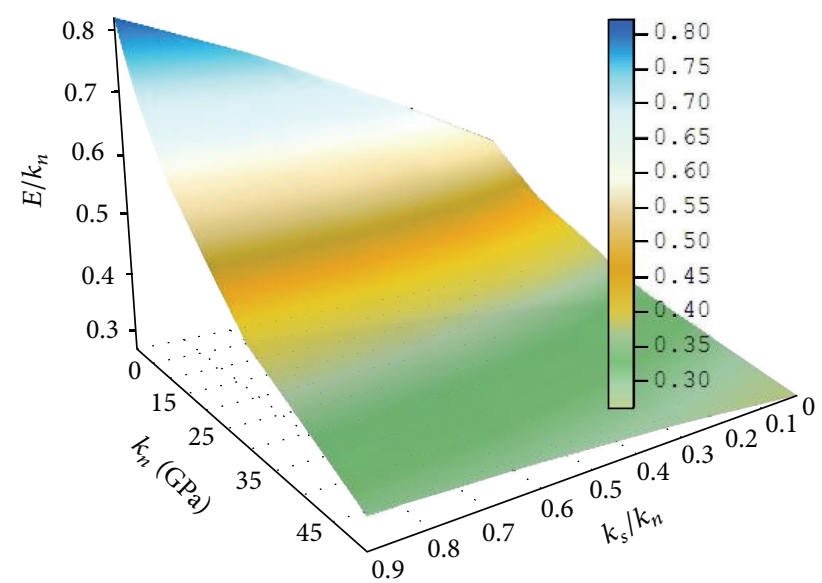

FIGURE 8: The changes in Young's modulus with the microdeformation parameters.

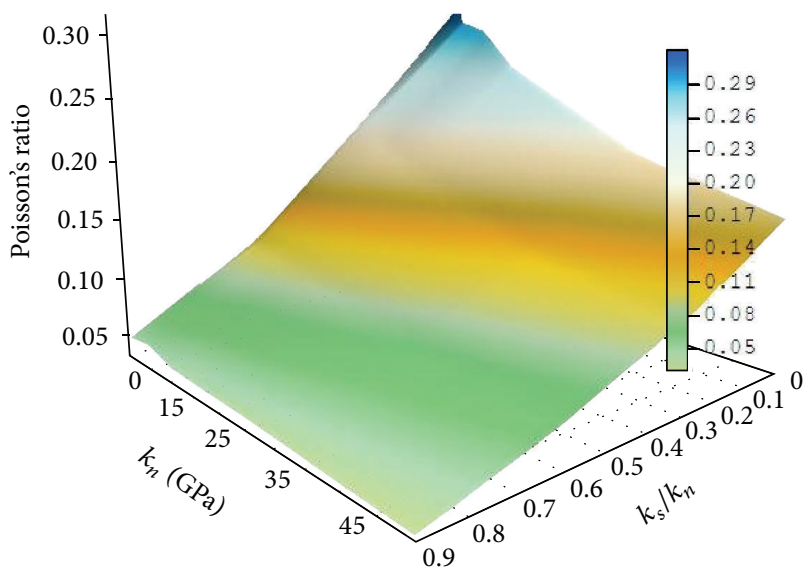

Figure 9: The changes in Poisson's ratio with the microdeformation parameters.

normal stiffness, Poisson's ratio is close to zero. When the stiffness ratio decreases, the lateral deformation increases. When the tangent stiffness is zero, Poisson's ratio reaches its maximum value. The relationship between Poisson's ratio and the stiffness ratio based on a series of numerical tests can be expressed using the following equation:

$$
\mu_{0}=0.1278 \eta^{2}-0.4061 \eta+0.3177
$$

where $\mu$ is Poisson's ratio and $\eta=k_{s} / k_{n}$ is the ratio of the tangent stiffness to the normal stiffness, which lies in the range $0 \sim 0.5$. Once $\eta$ is greater than 1.0, Poisson's ratio may be greater than 1.0 or negative, which disagrees with the properties of common solid media.

It should be noted that (10) does not consider the influence of the stiffness. If the stiffness is taken into consideration, Young's modulus and Poisson's ratio can be estimated with different normal stiffness as follows:

$$
\begin{gathered}
E=\left(0.2259 \eta-0.0058 k_{n}-0.0042 \eta k_{n}+0.5346\right) k_{n}, \\
\mu=-0.2760 \eta-0.0029 k_{n}+0.0031 \eta k_{n}+0.3200 .
\end{gathered}
$$

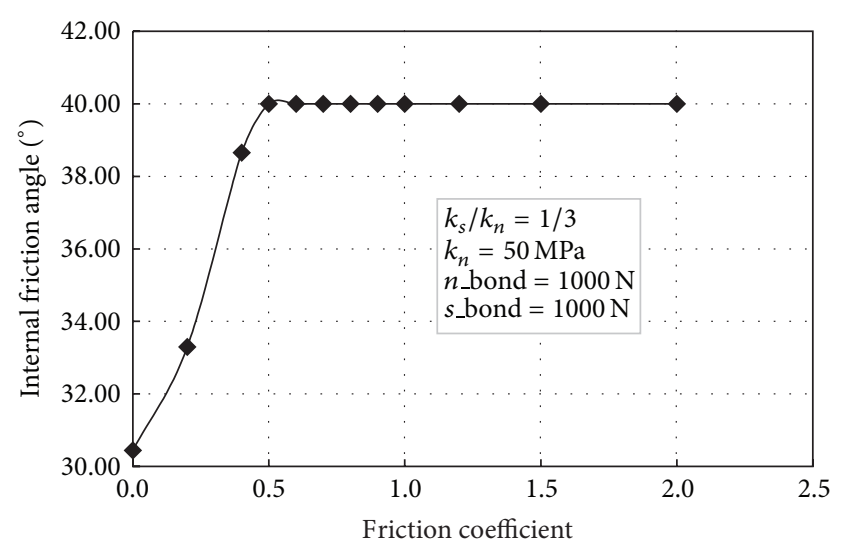

FIGURE 10: The changes in the internal friction angle at different coefficients of friction.

The changes in Young's modulus and Poisson's ratio with the changes in the microparameters are shown in Figures 8 and 9. The figures illustrate that the relationship between Young's modulus of an outwash deposit and the corresponding Poisson's ratio is not a simple linear relation but a double linear correlation in the calculation of the granular discrete element method. When the microparameters need to be determined, Young's modulus and Poisson's ratio can initially be assumed. Then, more reasonable values can be determined using (11). The steps should be repeated many times until Young's modulus and Poisson's ratio agree with the results of the macroscopic tests. In addition, the debris and stone blocks should be calibrated separately to obtain proper microparameters.

3.4. The Micromechanical Parameters of the Outwash Deposits. The normal contact force ( $n$-bond), tangent contact force (s_bond), and the coefficient of friction $\mu$ are the main microparameters related to macrostrength in a granular model based on PFC2D. These parameters correspond to the macrocohesion and the internal friction in the MohrCoulomb criterion. The coefficient of friction is the pure frictional force when the outwash deposit is completely destroyed. It determines the residual strength of the medium and can be approximately equal to the pure friction coefficient $\tan \varphi$ of the stone and debris mass.

As shown in Figure 10, when the coefficient of friction is low, the deformation of the stone and debris mass shows a linear variation. By contrast, when the friction coefficient is high, the deformation of the stone and debris mass shows a nonlinear variation. The internal friction angle of the medium first increases and then remains unchanged as the coefficient of friction increases. The cohesion shows similar behaviour, but it remains constant if the coefficient of friction is low. The cohesion will increase as the coefficient of friction increases, as shown in Figure 11.

It is not difficult to find that the microcoefficient of friction is sensitive to the macro-internal-friction angle. When the microparameters need to be determined, the bonding forces should initially be set to zero, then the friction 


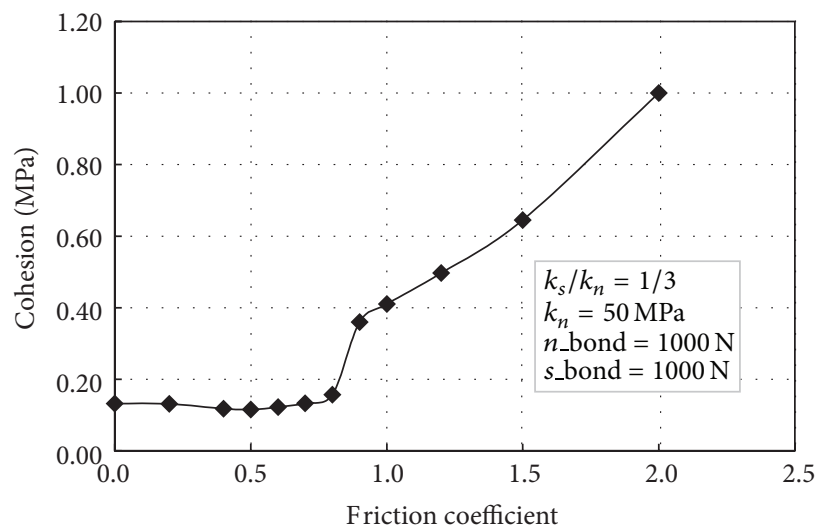

FIGURE 11: The cohesion remains constant if the coefficient of friction is low.

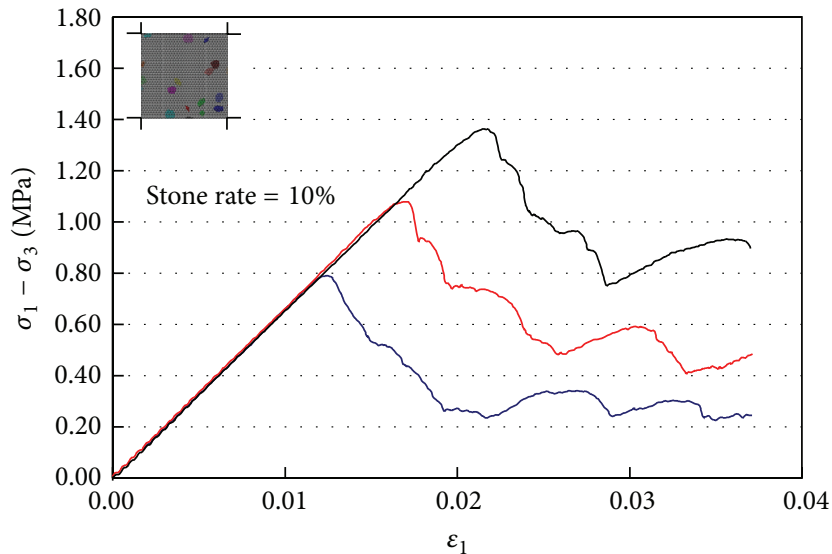

(a)

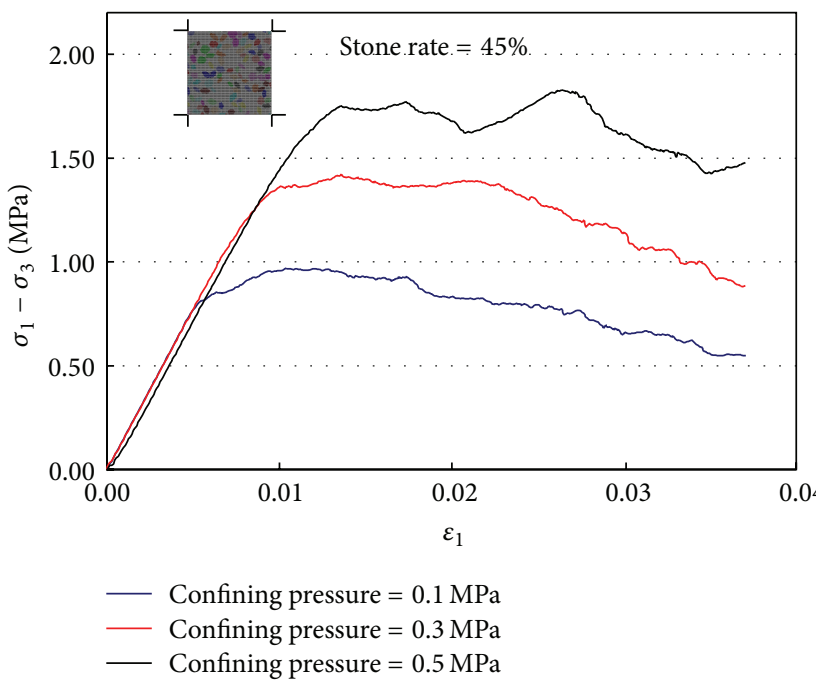

(c)

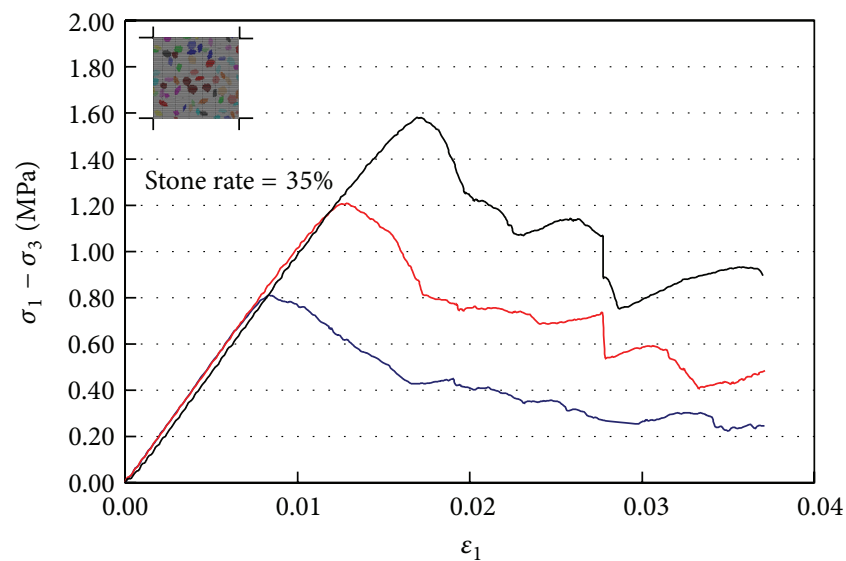

(b)

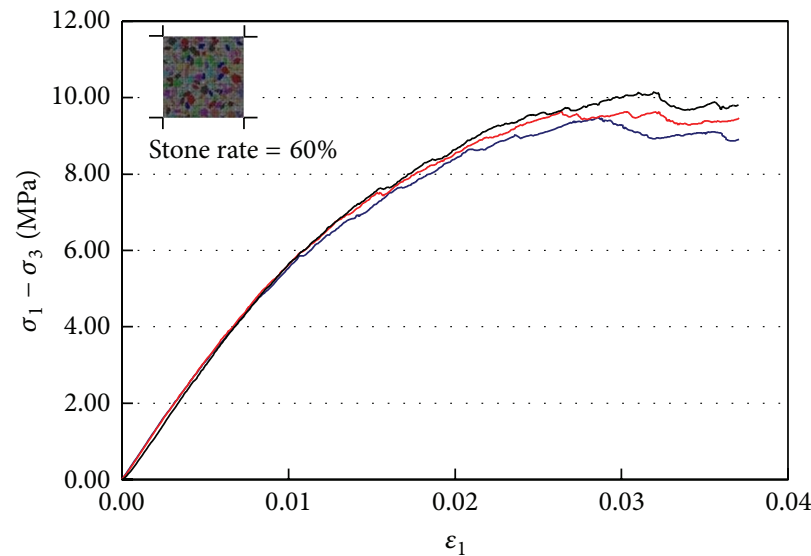

Confining pressure $=0.1 \mathrm{MPa}$

Confining pressure $=0.3 \mathrm{MPa}$

Confining pressure $=0.5 \mathrm{MPa}$

(d)

FIGURE 12: The biaxial compression curve at different confining pressures and stone block content. (a) Stone block content is 10\%; (b) stone block content is $35 \%$; (c) stone block content is $45 \%$; and (d) stone block content is $60 \%$. 
TABLE 1: The micromechanical parameters of outwash deposits.

\begin{tabular}{lcccccc}
\hline \multirow{2}{*}{ Material } & \multirow{2}{*}{ Density $\left(\mathrm{kg} / \mathrm{m}^{3}\right)$} & \multicolumn{2}{c}{ Bonding force $(\mathrm{N})$} & \multirow{2}{*}{ Stiffness ratio } & Normal stiffness (N/m) & \multirow{2}{*}{ Friction coefficient } \\
& & Normal & Tangential & & $1 e 8$ & 0.25 \\
Debris & 2200 & $1 e 3$ & $1 e 3$ & 0.0 & $9.5 e 9$ & 1.20 \\
Stone & 2700 & $2 e 6$ & $2 e 6$ & 0.2 & & \\
\hline
\end{tabular}

TABLE 2: The macromechanical parameters of outwash deposits.

\begin{tabular}{lcccc}
\hline Material & Young's modules $(\mathrm{MPa})$ & Poisson's ratio & Internal friction angel $\left(^{\circ}\right)$ & Cohesion $(\mathrm{MPa})$ \\
\hline Debris & 55.9 & 0.32 & 27.94 & 0.07 \\
Stone & 4950 & 0.20 & 56 & 12.0 \\
\hline
\end{tabular}

coefficient can be determined using trial calculations until the macromechanical properties are in line with the experimental results. Finally, the bonding forces are adjusted so that the parameters are consistent with the macromechanical properties of the outwash deposit.

When the bonding force is zero, the internal friction angle will reach its minimum value. Therefore, the determination of the internal friction angle should take the effect of the stiffness ratio into consideration. If the internal friction angle is unchanged when the coefficient of friction increases, the bonding force can be adjusted to be in agreement with the peak strength of the stress. In this work, the microparameters are shown in Table 1, and the corresponding macroparameters are shown in Table 2.

\section{The Analysis of the Mechanical Behaviour of the Outwash Deposits}

As a case study shown in Figure 1, the stone rate of outwash based on the statistical results is about $30 \%$ to $40 \%$, the mean length of long axis is about $4.13 \mathrm{~cm}$ with a variance 0.15 , and the mean axis ratio is 0.64 with a variance 0.17 . Then the random structure models of outwash deposits are generated according to the above information. Finally, numerical simulation and comparison are executed in order to analyse the effect of comprehensive mechanical behaviour.

\subsection{The Stress-Strain Curve Based on the Biaxial Compression} Test. The stress-strain curves from the numerical simulation tests of an outwash deposit under biaxial compression conditions can be divided into three stages: the elastic stage, the hardening stage, and the residual strength stage (shown in Figure 12). In the elastic stage, the deformation modulus is determined by the undamaged debris particles and stone block assemblies. As the deformation increases, fractures will first appear in the debris because of its low modulus and strength. When the outwash deposit enters into the hardening stage the debris particle bonding forces will fail gradually, and the deformation modulus will decrease slowly. However, the strength will increase continually due to the interaction force caused by mosaic effect between the debris particles and the stone blocks. When the stress reaches its peak strength, the outwash deposit will enter into the residual strength stage. The cementation ability between the debris particles is almost completely lost. The compressive-bearing capability of the sample cannot continue to increase. The compressive-bearing capability is controlled mainly by the interparticle friction. When the strain continues to increase, the stone blocks may rotate and tumble, which leads to the complete destruction of the structure of the outwash deposit. However, there is still some compression-bearing capability, which causes the residual strength of the outwash deposit. The residual strength shows fluctuations because of the contact action between the debris and the stone blocks.

As shown in Figure 13, the damage of a model with content ratio of stone block $40 \%$, length of long axis about 2 to $6 \mathrm{~cm}$, and the axial ratio 0.5 to 0.8 is compared in the loading process. It shows that cracks occur firstly between some blocks and then develop among the boundary of stone blocks and fill with the debris particles. The crack number will increase shapely with the strain increase until all bond contacts between debris particles are destroyed.

The curves for different debris bonding forces in Figure 14 show that the cementation strength of the debris particles decides the strength of outwash deposit and has a dominant role in the mechanical properties when the other conditions are fixed. With a stronger cementation force of debris particles, there is a smaller increase in the internal friction angle and a bigger increase for the cohesion. The mechanical properties of an outwash deposit with high cementation strength are more similar to the properties of the stone blocks. Conversely, if the cementation strength of the debris particles is weak, there is a bigger increase in the internal friction angle and a smaller increase in the cohesion. The mechanical properties of the outwash deposit are much closer to the elastic-plastic debris.

4.2. The Influence of the Aggregate Size. As shown in Figure 15, the larger the size of stone blocks is, the smaller the peak strength is. This situation can be interpreted by the perimeter ratio, which is defined as the ratio of all boundaries' perimeters of stone blocks and the model area. The perimeter ratio under the same stone ratio varies greatly. The smaller aggregate size has more stone blocks, so the perimeter ratio will reach a high level and form larger contact areas. The compressive strength also increases. When the number of stone blocks increases, the contact area of the debris and stone blocks and the interaction force between the debris and the 


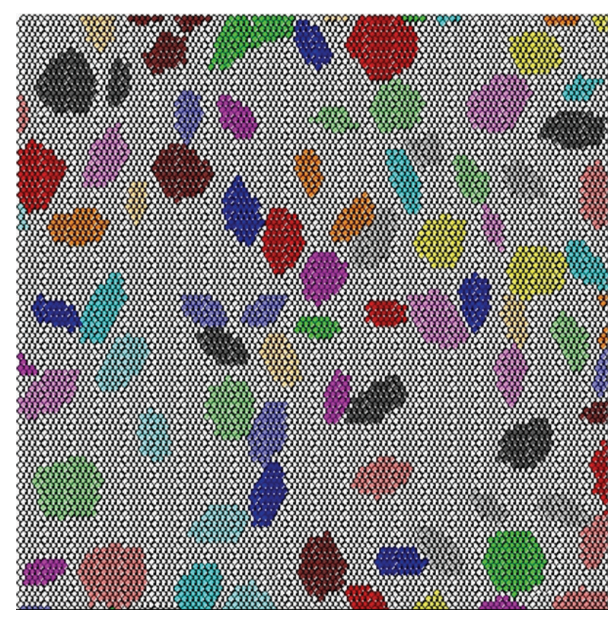

(a) Crk_num $=0$

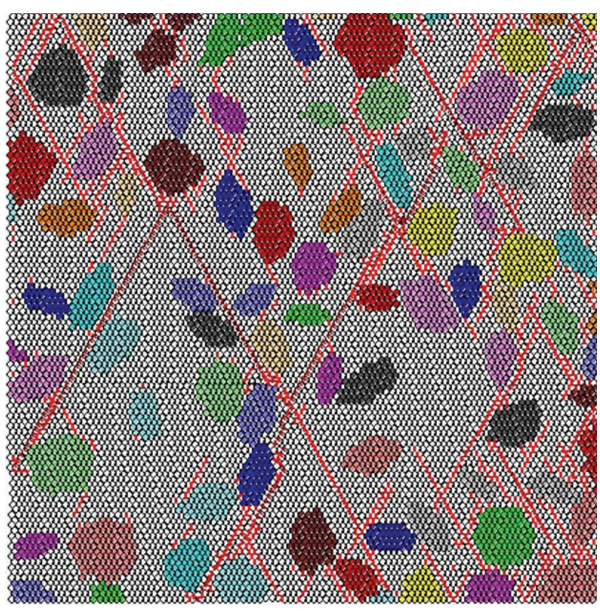

(c) Crk_num $=189$

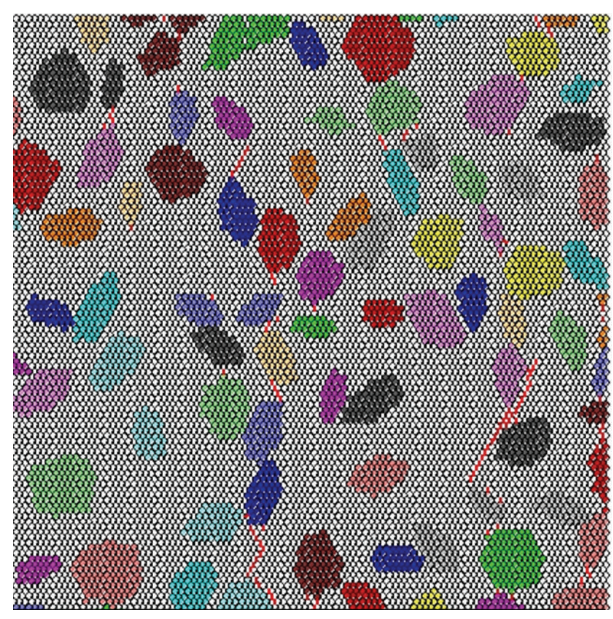

(b) Crk_num $=39$

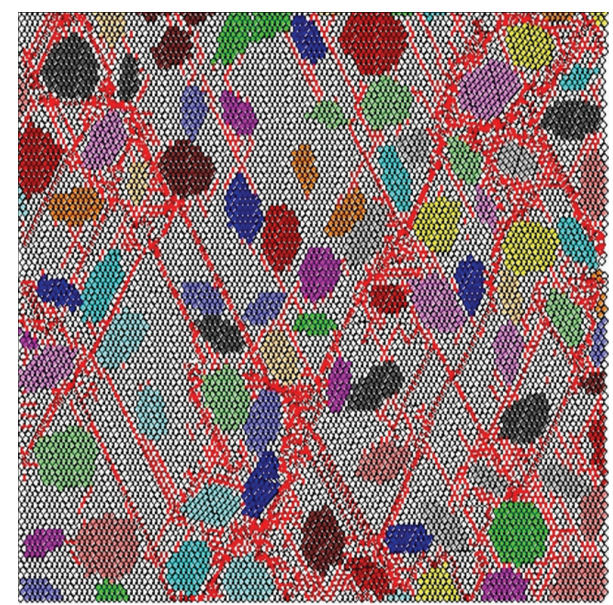

(d) Crk_num $=563$

FIGURE 13: The damage of outwash deposits with different stone block content; (a) shows a model with the initial content rate of stone block 40\%; (b) shows the cracks which first appear between some blocks; (c) shows fracture development among the boundary of stone blocks; and (d) shows the damaged outwash deposits.

stone blocks will increase, which just explains the reason why the macromechanical properties of the outwash deposit are improved with the number of stone blocks.

4.3. The Influence of the Stone Block Content. As shown in Figure 16, when there are few stone blocks in the outwash deposit, the debris-debris contact between particles will be the main contact type. The existence of stone blocks leads to the concentration of stress along their boundaries, which can aggravate the debris contacts to damage; the peak compressive strength keeps close to the pure debris with slight fluctuation. Meanwhile, due to the stress concentration, the outwash deposits become more brittle. The shear strength is decreased. Many experiments show that when the content of stone blocks is less than $25 \%$, the characteristic is more close to pure debris. By contrast, when the content of stone blocks is greater than $25 \%$, there is not only debris-stone contact in the outwash deposits but also stone-stone contact.
The compressive and shear strengths are improved. When the content of stone blocks is more than 50\%, the main contact type in the outwash deposits is stone-stone. The mechanical properties are better in stone-filled materials. The compressive and shear strengths are significantly improved in the stone-filled materials.

However, when the stress-strain curves enter the residual strength stage, there is an obvious fluctuation after the peak of the curve, the amplitude of which is related to the size and content ratio of stone blocks, so in order to study the macroshear strength, the peak value of stress-strain curves is used to calculate shear property.

4.4. The Macroparameter Analysis of the Outwash Deposits. In terms of the stress-strain curves, for any content rate of stone block, cementation strength, and block size, the major factor that influences the mechanical properties of the 


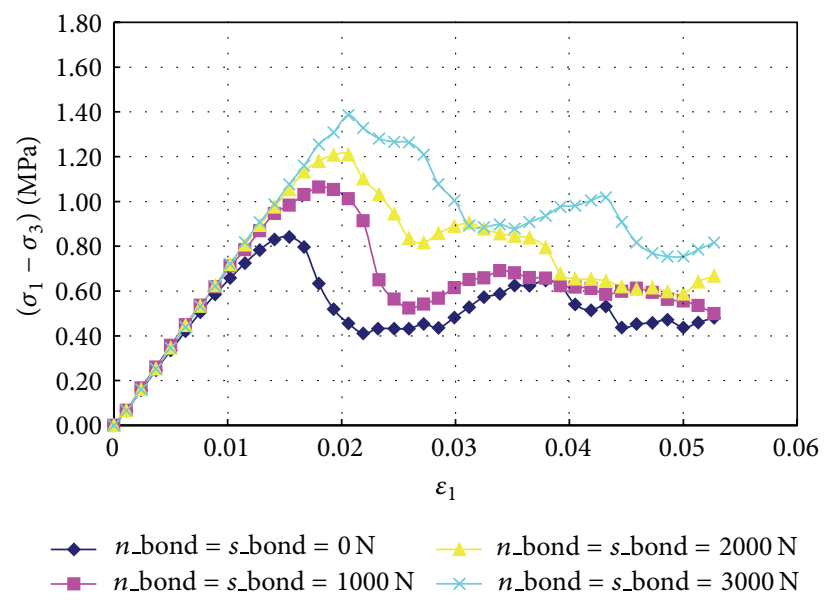

FIGURE 14: The biaxial compression curves with different debris bonding force.

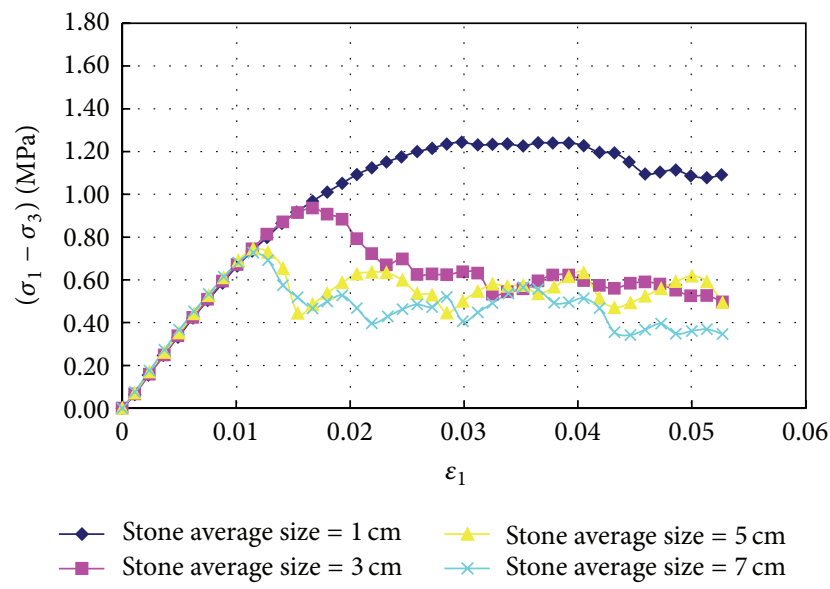

Figure 15: The biaxial compression curves with different average sizes of stone blocks.

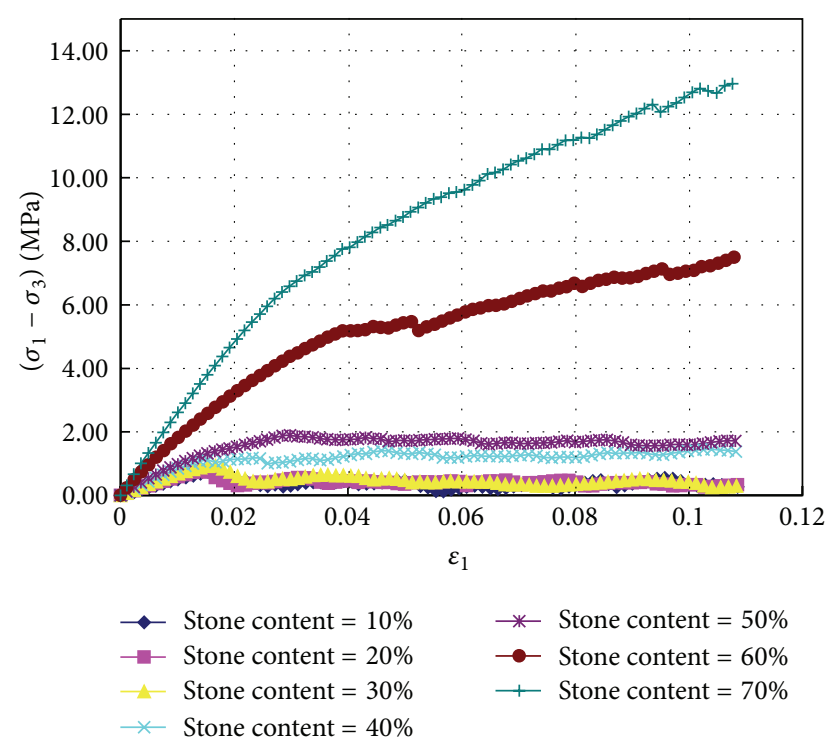

FIGURE 16: The biaxial compression curve with different stone block contents.

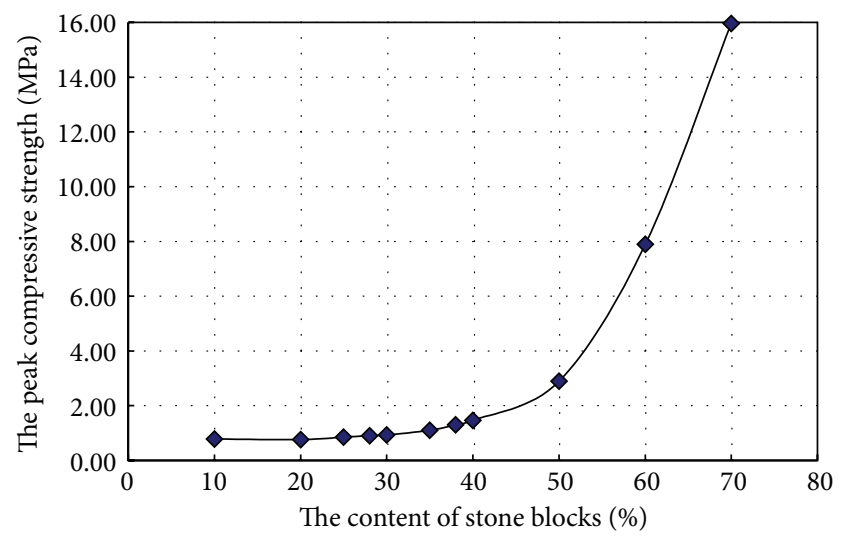

FIGURE 17: The peak compressive strength with different stone block contents.

outwash deposits can be attributed to the dominant contact type, which is defined as the ratio of some particle contact number and total contact number in numerical models. The particle contact can be divided into three kinds, respectively, the debris-debris, debris-stone, or stone-stone contact.

If ratio of stone block is high enough to make stone-stone contact becoming dominant contact type, the characteristic of outwash deposit is close to rockfill. For the nature medium, several kinds of contacts exist at that time, which will affect the comprehensive mechanical property greatly. Although the different geometries and distributions also affect the mechanical properties, the content of stone blocks is the decisive factor. For the different stone block contents, the peak strength under the confining pressure of $1.5 \mathrm{MPa}$ can be calculated approximately using formula (12) as a cubic parabola curve (shown in Figure 17):

$$
P_{k}=141.84 \alpha^{3}-90.44 \alpha^{2}+16.00 \alpha+0.2894
$$

where $\alpha$ is the content ratio of stone blocks and $P_{k}$ is the peak strength.

It should be noted that the compressive strength of the outwash deposit increases slowly when content ratio of stone 


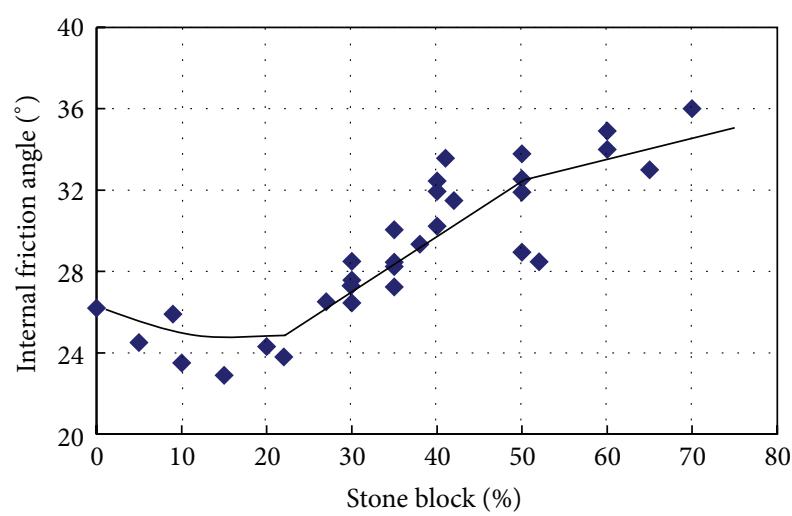

(a)

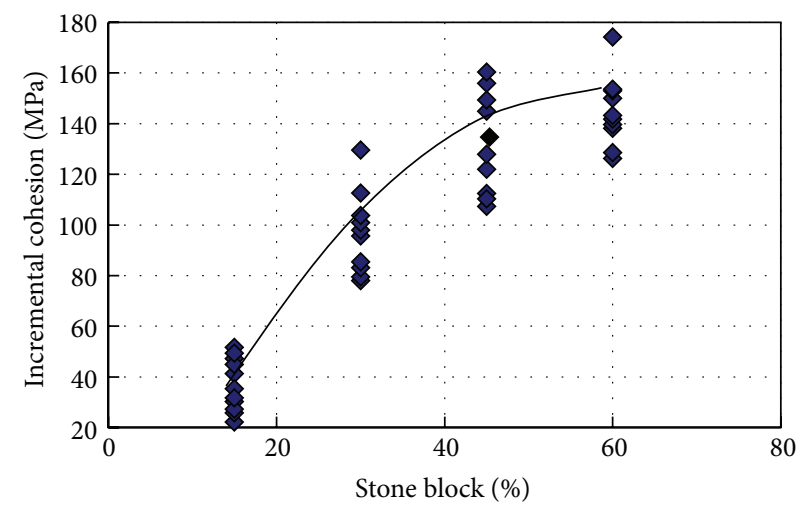

(b)

FIGURE 18: Changes of shear strength of outwash deposits with different contents and distributions of stone blocks; (a) shows changes of the internal friction of outwash deposit; (b) shows changes of the cohesion of outwash deposit.

blocks lies between 25 and 50\%. Once the content of stone blocks is greater than $50 \%$, the stone-stone contact becomes the dominant factor, which causes the compressive strength to increase sharply. The internal friction angle versus the stone block content can be described using three stages, as shown below:

$$
\varphi= \begin{cases}180.9393 \alpha^{2}-49.5165 \alpha+26.5026, & \alpha<25 \%, \\ 19.4271 \alpha+22.1719, & 25 \%<\alpha<50 \%, \\ 23.4286 \alpha+19.1429, & \alpha>50 \%,\end{cases}
$$

where $\varphi$ is the internal friction angle of the outwash deposit and $\alpha$ is the stone block content.

Furthermore, it can be observed from Figure 18(a) that the fluctuation of the internal friction angle is approximately $0 \sim 8^{\circ}$ for the same stone blocks content, which agrees with the fluctuations in the stress-strain curves. By contrast, the cohesion of outwash deposit increases clearly with the stone block content due to the interaction force between stone blocks as shown in Figure 18(b). The amplitude of variation will increase significantly with the content of stone blocks. However, it is usually difficult to quantify the amplitude due to roughness, size, characteristic of stone block, and so on.

Therefore, for outwash deposits with extremely uneven shapes and distributions of stones, the compressive strength and shear strength are primarily controlled by the stone block content. The randomness of the stone block distributions causes large deviations in the mechanical parameters. For example, the internal friction angle may have a large fluctuation at the same stone block content. This feature is the cause of the poor results of the geotechnical experiments.

\section{Discussions}

Outwash deposits are a type of debris-stone mixture. The mechanical properties of these deposits are affected by the composition of the debris and stone blocks. As the debris cementation increases, the compressive strength increases. However, the internal friction angle has a negative correlation with the cementation force. The stone block content is the decisive factor for the mechanical properties of outwash deposits. The randomness of the structural composition leads to significant fluctuations in the properties, which also creates large discrepancies in the mechanical parameters.

The major influences of the particle sizes, geometric distribution, and stone block content are to determine the contact type in the outwash deposits. When the debrisdebris contact is the dominant contact type, there is little influence from the stone blocks on the mechanical properties of the outwash deposits. When the debris-stone contact is the dominant contact type, the stone block content has a positive influence on the compressive and shear strengths. When the stone-stone contact is the dominant contact type, the roles of the debris and stone blocks are reversed and the properties of the outwash deposits are much closer to a rock-filled material.

Although it is difficult to determine the mechanical parameters of an outwash deposit using indoor experiments, the properties of the medium can still be researched using indirect approaches. The mechanical parameters of the debris and stone blocks can be determined from the indoor experiments. Then, the biaxial compression numerical experiment using the granular discrete element method can be carried out to analyse the influence of the stone blocks on the properties of outwash deposits, which makes up for the limitation that the indoor experiments cannot consider the large stone blocks and offers a beneficial supplement to aid in the geological parameter determination.

\section{Conclusions}

According to the grain size and shape statistics from field statistics, the numerical granular discrete element model with randomised generation of stone blocks can be used with hypothesis that the length of the long axis and the axial ratio all follow normal distributions and the azimuth of the long axis follows uniform distribution in $0 \sim 360^{\circ}$. The relationships between the macro- and micromechanical parameters are discussed and the mechanical behaviour of the outwash 
deposit is affected by the cementation strength, the size of stone blocks, the stone block content, and the distribution of the stone blocks. These properties are analysed so that they can be better understood by the geologists working on the democratisation of the Engineering Project.

\section{Conflict of Interests}

The authors declare that there is no conflict of interests regarding the publication of this paper.

\section{Acknowledgments}

The work was supported by the National Natural Science Foundation of China (no. 51309089 and no. 51379065), the National Key Technology R\&D Program (no. 2013BAB06B00), the National Basic Research Program of China (973 Program) (no. 2015CB057903), the Natural Science Foundation of Jiangsu Province (no. BK20130846) and the Fundamental Research Funds for the Central Universities (no. 2014B04914).

\section{References}

[1] W. G. Holtz and H. J. Gibbs, "Triaxial shear tests on pervious gravelly soils," Journal of the Soil Mechanics and Foundations Division, vol. 82, no. 1, pp. 1-22, 1956.

[2] R. J. Chandler, "The inclination of Talus, Arctic Talus Terraces, and other slopes composed of granular materials," The Journal of Geology, vol. 81, no. 1, pp. 1-14, 1973.

[3] X. H. You, Stochastic structural model of the earth-rock aggregate and its application [Ph.D. thesis], Beijing Jiaotong University, Beijing, China, 2001.

[4] X. H. You and J. S. Tang, "Research on horizontal push-shear in-situ test of debris and rock-mixture," Chinese Journal of Rock Mechanics and Engineering, vol. 21, no. 10, pp. 1537-1540, 2002.

[5] X. H. You, G. He, and X. Li, "Micro-handling technology of earth-rock aggregate slope," Hydrogeology and Engineering Geology, vol. 30, no. 1, pp. 18-21, 2003.

[6] J. M. He, Study of deformation and failure mechanisms of rockdebris aggregate in three gorges reservoir area [Ph.D. thesis], China University of Mining and Technology, Beijing, China, 2004.

[7] X. Li, Q. L. Liao, J. M. He, and J. Chen, "Study on in-situ tests of mechanical characteristics on soil-rock aggregate," Chinese Journal of Rock Mechanics and Engineering, vol. 26, no. 12, pp. 2377-2384, 2007.

[8] T. Lebourg, J. Riss, and E. Pirard, "Influence of morphological characteristics of heterogeneous moraine formations on their mechanical behaviour using image and statistical analysis," Engineering Geology, vol. 73, no. 1-2, pp. 37-50, 2004.

[9] T. R. Reid and J. P. Harrison, "A semi-automated methodology for discontinuity trace detection in digital images of rock mass exposures," International Journal of Rock Mechanics and Mining Sciences, vol. 37, no. 7, pp. 1073-1089, 2000.

[10] Z. Q. Yue, S. Chen, and L. G. Tham, "Finite element modeling of geomaterials using digital image processing," Computers and Geotechnics, vol. 30, no. 5, pp. 375-397, 2003.

[11] Z. Q. Yue, S. Chen, H. Zheng, and G. H. Tang, "Digital image proceeding based on finite element method for geomaterials,"
Chinese Journal of Rock Mechanics and Engineering, vol. 23, no. 6, pp. 889-897, 2004.

[12] Q. L. Liao, X. Li, W. C. Zhu, and J. S. Liu, "Structure model construction of rock and soil aggregate based on digital image technology and its numerical simulation on mechanical structure effects," Chinese Journal of Rock Mechanics and Engineering, vol. 29, no. 1, pp. 155-162, 2010.

[13] W. J. Xu, R. L. Hu, Z. Q. Yue, and R. J. Tan, "Mesostructural character and numerical simulation of mechanical properties of soil-rock mixtures," Chinese Journal of Rock Mechanics and Engineering, vol. 26, no. 2, pp. 300-311, 2007.

[14] W. J. Xu, Z. Q. Yue, and R. L. Hu, "A current status of digital image based quantitative analysis of internal structures of debris, rock and concretes and associated numerical simulation," Journal of Engineering Geology, vol. 15, no. 3, pp. 289-313, 2007.

[15] W. J. Xu, R. L. Hu, Z. Q. Yue, R. Zhang, and G. L. Wang, "Research on relationship between rock block proportion and shear strength of soil-rock mixtures based on digital image analysis and large direct shear test," Chinese Journal of Rock Mechanics and Engineering, vol. 27, no. 5, pp. 996-1007, 2008.

[16] P. A. Cundall and O. D. L. Strack, "A discrete numerical model for granular assemblies," Geotechnique, vol. 29, no. 1, pp. 47-65, 1979.

[17] Itasca Consulting Group, PFC2D (Particle Flow Code in 2 Dimensions) (Version 3.1), Itasca Consulting Group, Minneapolis, Minn, USA, 2004.

[18] J. Yoon, "Application of experimental design and optimization to PFC model calibration in uniaxial compression simulation," International Journal of Rock Mechanics and Mining Sciences, vol. 44, no. 6, pp. 871-889, 2007.

[19] Y. C. Wang and P. Mora, "Macroscopic elastic properties of regular lattices," Journal of the Mechanics and Physics of Solids, vol. 56, no. 12, pp. 3459-3474, 2008. 

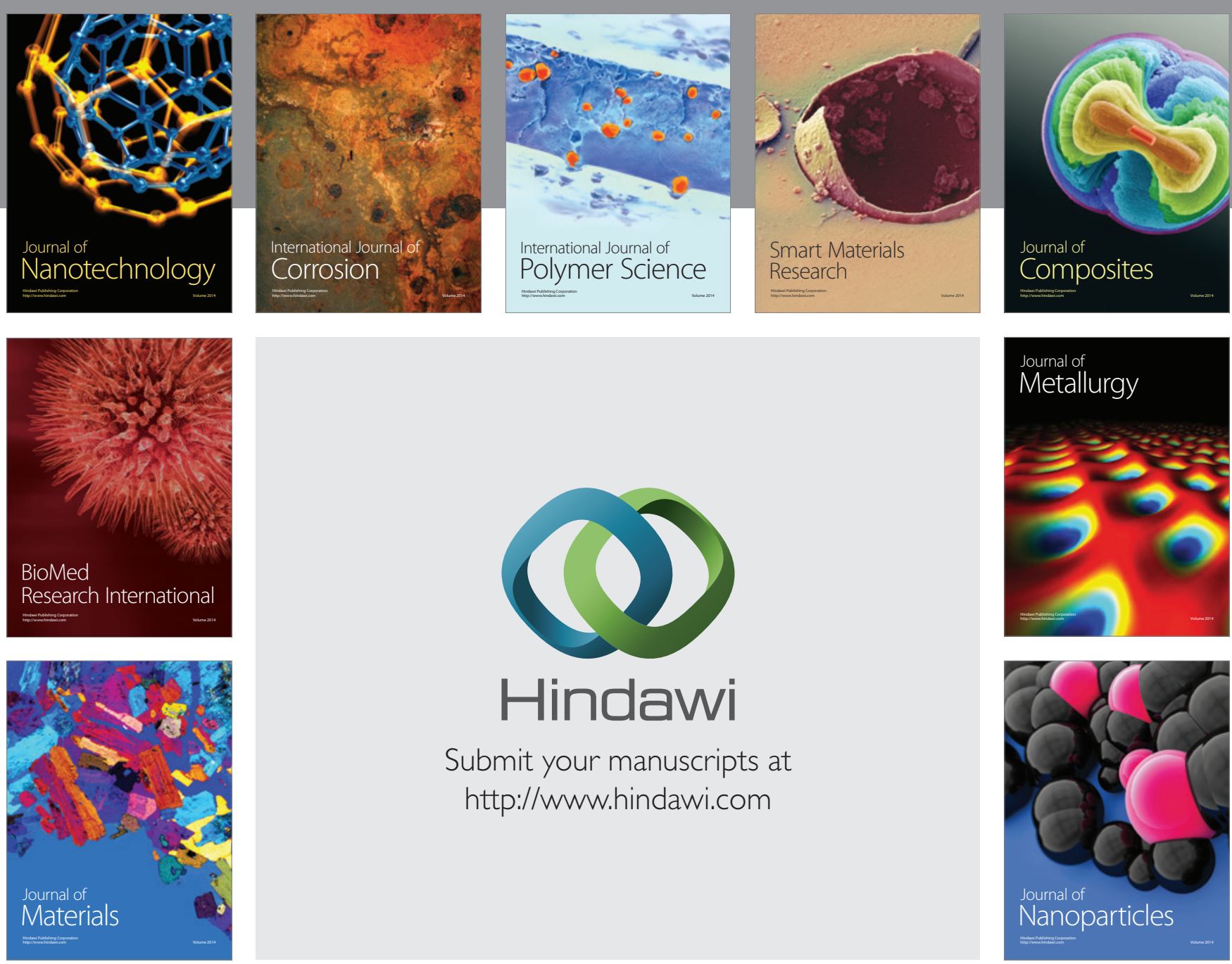

Submit your manuscripts at http://www.hindawi.com
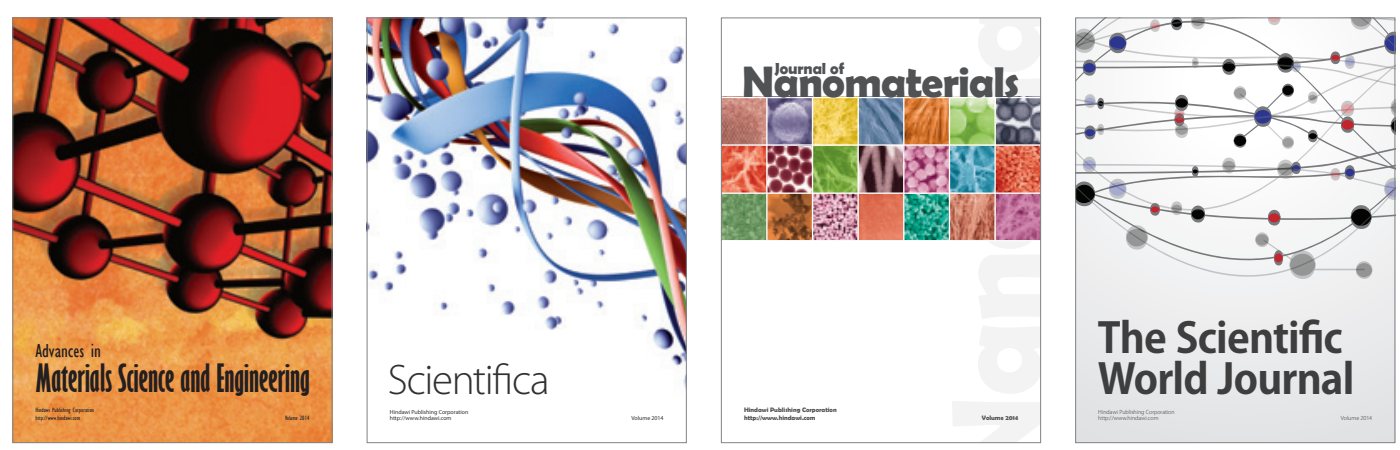

\section{The Scientific World Journal}
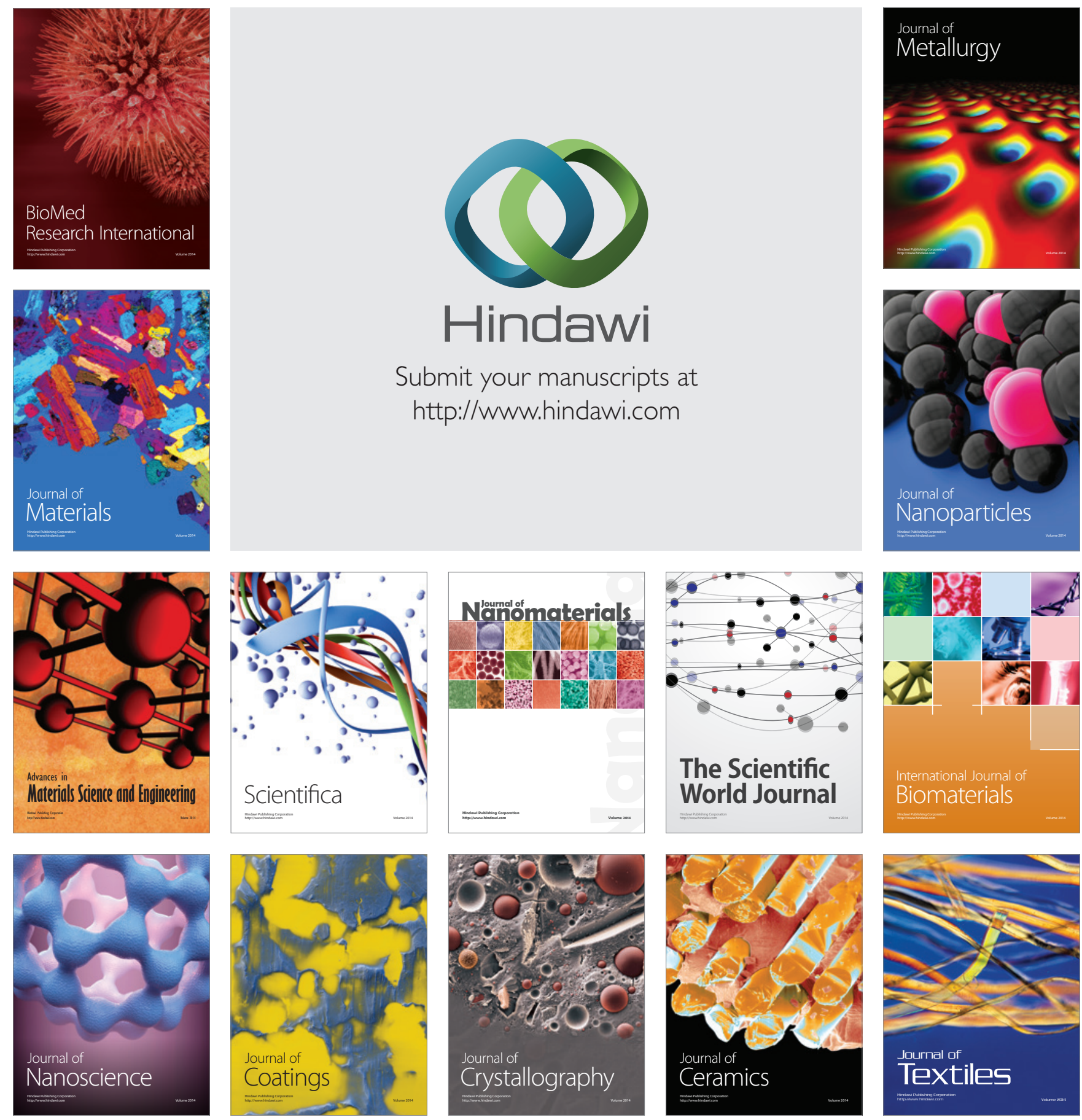